\title{
Transport and magnetic properties of NdCuGe compound
}

\author{
V. Goruganti, ${ }^{1}$ K. D. D. Rathnayaka, ${ }^{1}$ Joseph H. Ross, Jr., ${ }^{1, a)}$ Y. Öner, ${ }^{2}$ C. S. Lue, ${ }^{3}$ \\ and Y. K. Kuo ${ }^{4}$ \\ ${ }^{1}$ Department of Physics, Texas A\&M University, College Station, Texas 77843-4242, USA \\ ${ }^{2}$ Department of Physics, Faculty of Sciences and Letters, Istanbul Technical University, \\ 34469 Maslak, Istanbul, Turkey \\ ${ }^{3}$ Department of Physics, National Cheng Kung University, Tainan 70101, Taiwan \\ ${ }^{4}$ Department of Physics, National Dong Hwa University, Hualien 97401, Taiwan
}

(Received 22 December 2007; accepted 6 February 2008; published online 11 April 2008)

\begin{abstract}
Electrical resistivity, heat capacity, and dc magnetization measurements were performed on the $\mathrm{AlB}_{2}$-type intermetallics $\mathrm{NdCuGe}$ and $\mathrm{LaCuGe}$ over the temperature range of $2-300 \mathrm{~K}$. NdCuGe exhibits magnetization attributable to $\mathrm{Nd}^{3+}$ moments, with an antiferromagnetic (AF) transition at 3.1 K. Magnetization, heat capacity, and resistivity measurements were used to determine the crystalline electric field (CEF) splitting of the $\mathrm{Nd}^{3+}$ magnetic sublevels. The ground state is a doublet composed of $\pm 5 / 2$ levels and the $\mathrm{AF}$ state is built from this magnetic doublet, with a reduction in the average sublattice magnetization attributable to site disorder among $\mathrm{Cu}-\mathrm{Ge}$ sites. Low-temperature electrical transport is dominated by spin-disorder scattering among CEF-split levels and a small $T^{2}$ spin fluctuation term. The latter is nearly two orders of magnitude smaller than previously observed in isostructural $\mathrm{CeCuGe}$, for which the anomalous observed thermopower exponent was attributed to extended spin fluctuations above the magnetic transition. By contrast, simple-metallic behavior provides a good fit to the $\mathrm{NdCuGe}$ thermopower, thus, the $\mathrm{CeCuGe}$ results can be attributed to resonant scattering due to partial hybridization of the localized orbitals. (C) 2008 American Institute of Physics. [DOI: 10.1063/1.2904856]
\end{abstract}

\section{INTRODUCTION}

As is well known, rare-earth based compounds exhibit a variety of magnetic behavior depending on the ratio of exchange interactions to crystal field interactions. Of these compounds, ternary equiatomic lanthanide silicides and germanides with the general formula $R T S i$ and $R T G e$ ( $R=$ rare earth; $T=$ transition metal), which crystallize in several different structure types, have been extensively studied. ${ }^{1-3}$ In such materials, the competition between exchange interactions and crystal field splitting can give rise to complex magnetic structures and to unusual magnetization processes. For the 111 systems referred to above, having hexagonal crystal structures, the hybridizations between $f$ electrons and $d$ electrons are relatively weak due to a large separation between $R^{3+}-R^{3+}$ atoms in the lattice. The Ruderman-Kittel-KasuyaYosida-type interaction is generally the dominant mechanism for magnetic ordering. In spite of numerous experimental studies of these compounds, there are limited reports for the $R \mathrm{CuGe}$ system. We have recently carried out magnetization, resistivity, and thermal transport measurements on $\mathrm{CeCuGe}$, crystallizing in the hexagonal $\mathrm{AlB}_{2}$-type structure. ${ }^{4}$ This compound is found to be quite magnetically soft, with a very small magnetocrystalline anisotropy, contrary to what was reported in a previous investigation. ${ }^{5}$ In this study, we present magnetic, thermal, and transport properties of $\mathrm{NdCuGe}$, shown to have an antiferromagnetic (AF) transition at $3.4 \mathrm{~K}$ by neutron scattering measurements. ${ }^{6}$ Although the crystallographic structures of these compounds are identical, their electronic and thermal transport properties are

${ }^{a)}$ Electronic mail: jhross@tamu.edu. quite different in some respects. For example, while $\mathrm{CeCuGe}$ exhibits a ferromagnetic transition at $T_{C}=10 \mathrm{~K}$, strongly influenced by spin fluctuations, ${ }^{4} \mathrm{NdCuGe}$ has its $\mathrm{AF}$ transition at $3.4 \mathrm{~K}$ associated with the alignment of crystal-field-split levels of the magnetic $\mathrm{Nd}$ ion, and transport properties much closer to those of a classic metallic system.

\section{EXPERIMENTAL}

Samples of $\mathrm{NdCuGe}$ and $\mathrm{LaCuGe}$ were prepared for this study by arc melting the elemental constituents under argon atmosphere, using starting materials of $99.9 \%$ purity. Ingots were remelted several times. To ensure a homogeneous final material, samples were annealed at $800^{\circ} \mathrm{C}$ for two months. The structure was analyzed by $\mathrm{x}$-ray diffraction using $\mathrm{Cu} K \alpha$ radiation. Both samples were found to be single phase with the $\mathrm{AlB}_{2}$ structure (space group designation $P 6 / \mathrm{mmm}$, No. 191), with mixed occupation of $\mathrm{Cu}$ and $\mathrm{Ge}$ on $\mathrm{B}$ sites in the $\mathrm{AlB}_{2}$ lattice, as reported elsewhere. ${ }^{6,7}$ Lattice constants from the x-ray fit for $\mathrm{NdCuGe}$ were found to be $a=0.4277 \mathrm{~nm}$ and $c=0.3881 \mathrm{~nm}$, in good agreement with what has been reported in the literature.

A superconducting quantum interference device (SQUID) magnetometer was used to measure the dc magnetization in the temperature range of $5-300 \mathrm{~K}$. The magnetic field was applied along the long axis of the sample in order to minimize the demagnetizing field. The temperature dependence of the heat capacity $(C)$ was measured using an adiabatic method down to $2 \mathrm{~K}$, using a Quantum Design PPMS system.

Resistivity was measured with a standard dc four-probe setup over a 4-300 K temperature range. A calibrated Ge 


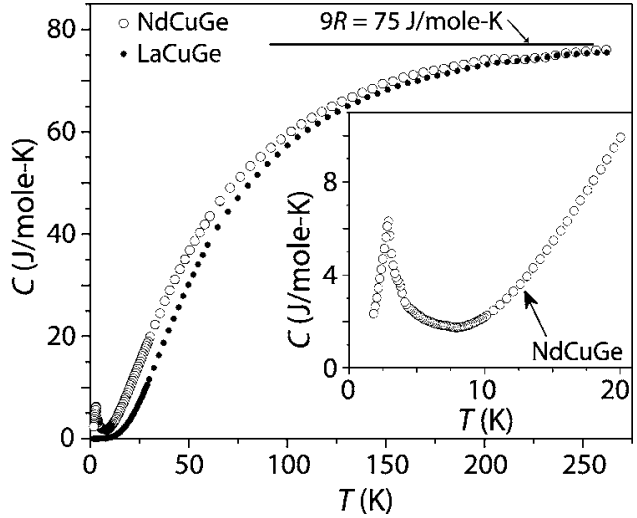

FIG. 1. Heat capacity for $\mathrm{NdCuGe}$ and for nonmagnetic $\mathrm{LaCuGe}$. Inset: low-temperature portion of $\mathrm{NdCuGe}$ data showing sharp feature at $T_{N}$.

thermometer (GR-200A-2500) was used to measure the temperature below $80 \mathrm{~K}$. For higher temperatures, a calibrated Pt thermometer was used. Electrical contacts were made using silver paint and $25 \mu \mathrm{m}$ gold wire.

The NdCuGe ingot was cut to a rectangular parallelepiped with an approximate size of $1.5 \times 1.5 \times 5.0 \mathrm{~mm}^{3}$ for the thermoelectric power $(S)$ measurements. All experiments were performed while warming, at a rate less than $20 \mathrm{~K} / \mathrm{h}$. The reproducibility of the measurements was better than $2 \%$. More details about the technique and measurements were given in Ref. 8.

\section{RESULTS AND DISCUSSION}

\section{A. Heat capacity measurements}

Figure 1 shows heat capacity results for both $\mathrm{NdCuGe}$ and $\mathrm{LaCuGe}$ for temperatures of up to $250 \mathrm{~K}$. At high temperatures, both curves approach the classical value $3 R \times N$ due to vibrational modes of the $N=3$ atoms/f.u., as seen in the figure. For the isomorphous nonmagnetic compound $\mathrm{LaCuGe}$, the curve monotonically varies, with no anomaly observed down to $1.8 \mathrm{~K}$. Below $10 \mathrm{~K}$, the LaCuGe heat capacity could be fitted to the equation $C=\gamma T+\beta T^{3}$, with electronic coefficient $\gamma=2.22 \mathrm{~mJ} / \mathrm{mole}^{2}$ and $\beta$ corresponding to a Debye temperature $\left(\Theta_{D}\right)$ equal to $231 \mathrm{~K}$ in the low- $T$ limit. After subtracting the electronic contribution $\gamma T$, we also fit the full temperature dependence to a Debye model according to established procedure. ${ }^{9}$ In this fit, the effective $\Theta_{D}$ rises to a low-temperature peak of $280 \mathrm{~K}$ near $T=10 \mathrm{~K}$, followed by a minimum of $\Theta_{D}=240 \mathrm{~K}$ near $30 \mathrm{~K}$, and a broad maximum of $\Theta_{D} \approx 260 \mathrm{~K}$ near $100 \mathrm{~K}$. The steplike feature centered about approximately $3.1 \mathrm{~K}$ for $\mathrm{NdCuGe}$ (inset) corresponds to the AF transition previously identified, while the difference between the $\mathrm{NdCuGe}$ and $\mathrm{LaCuGe}$ curves at higher temperatures can be attributed to the effect of crystalline electric field (CEF) splitting of the $\mathrm{Nd}$ magnetic levels.

We isolated the magnetic contribution to the heat capacity $\left(\Delta C_{\text {mag }}\right)$ by subtracting an interpolated $\mathrm{LaCuGe}$ curve from that of $\mathrm{NdCuGe}$. In doing so, temperatures for $\mathrm{LaCuGe}$ were scaled proportional to $\sqrt{M_{\mathrm{LaCuGe}}} / M_{\mathrm{NdCuGe}}$, where the terms in the ratio are molar masses. (A method utilizing partial Debye temperatures ${ }^{10}$ gives nearly identical results, as



FIG. 2. (Color online) Magnetic contribution to the heat capacity of $\mathrm{Nd}$ $\mathrm{CuGe}$ at zero field, normalized to the ideal gas constant. Solid curve shows Schottky-anomaly fit for crystal-field-split levels as described in the text. Splitting of these levels is schematically shown below the data. Inset: magnetic entropy extracted from the heat capacity.

does simply using data uncorrected for the masses.) The resulting $\Delta C_{\text {mag }}$ is shown in Fig. 2; above the low-temperature magnetic transition region, a Schottky-like anomaly is seen due to the freezing out of CEF-split magnetic levels. From $\left(\Delta C_{\text {mag }}\right)$, we obtained the magnetic entropy using the relation $S_{\text {mag }}=\int\left(\Delta C_{\text {mag }} / T\right) d T$, with the result shown in the inset of Fig. 2. (There is a deficit in computed $S_{\text {mag }}$ due to the $1.8 \mathrm{~K}$ measurement limit; a power-law extrapolation of the lowest few $\Delta C$ values below $1.8 \mathrm{~K}$ indicates this deficit to be approximately $0.1 R$. This small additive term has not been included in the plot of Fig. 2.) $S_{\text {mag }}$ reaches a plateau at high temperatures, very close to the total magnetic entropy $S / R$ $=\ln (2 J+1)=2.30$ for $J=9 / 2$, as shown by the horizontal arrow in the inset figure. This result is consistent with the expected $J=9 / 2$ state for trivalent $\mathrm{Nd}$ and it provides additional confidence in the subtraction of the lattice and electronic parts of $C$. As can be seen from the inset, $S_{\text {mag }}$ has its largest drop over the temperature range corresponding to the Schottky-like peak in $\Delta C_{\text {mag }}$ due to CEF splitting, while a further drop is observed near $T_{N}$.

$\mathrm{Nd}$ levels within the point-group symmetry of $\mathrm{NdCuGe}$, including atomic disorder on the $\mathrm{Ge}$ and $\mathrm{Cu}$ sites, may be split into five separate Kramers doublets, ${ }^{11}$ so we derived a theoretical expression for the corresponding heat capacity, allowing the energies of the five doublets to vary arbitrarily in a least-square fit to $\Delta C_{\text {mag }}(T)$ above $17 \mathrm{~K}$. This relationship provided an excellent fit to the data, as shown in the solid curve in the main plot of Fig. 2. The fitting result, with levels schematically shown in Fig. 2, corresponds to a doubly degenerate ground state with quadruplets at energies of 65 and $146 \mathrm{~K}$ relative to the ground state. Thus, despite the $\mathrm{Ge}$ and $\mathrm{Cu}$ site disorder, the fitted level multiplicity is the same as for the sixfold axial symmetry of the hexagonal virtual lattice, for which two quadruplets and one doublet would be expected.

The drop in entropy near $T_{N}$ is close to that expected for a two-level ground state, although the theoretical value $\Delta S / R=\ln (2)=0.69$ is not reached until a temperature of 9.1 K, corresponding to the extent of the visible tail in Fig. 2. Similarly, the jump in $\Delta C_{\text {mag }}$ at $T_{N}$ is much smaller than the value $1.5 R$ expected for a two-level ground state. ${ }^{12}$ Although 


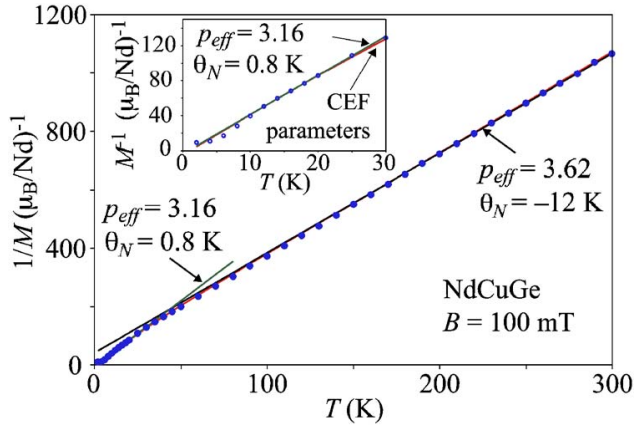

FIG. 3. (Color online) Inverse magnetization for NdCuGe measured in a DC field of $100 \mathrm{mT}$. Straight lines: Curie-Weiss fits with effective moments $3.16 \mu_{B}$ at low temperatures and $3.62 \mu_{B}$ at high temperatures, as labeled. Solid curve: calculated result obtained using CEF-split levels. Inset: expanded view at low temperature, with high-temperature Curie-Weiss fit omitted for clarity.

fluctuations will normally reduce this value, the present result is considerably smaller than observed, for example, in $\mathrm{Nd}_{2} \mathrm{Ni}_{2} \mathrm{~Pb}$ (Ref. 13) or a series of commensurate-AF $\mathrm{Gd}$ compounds. ${ }^{10}$ The enhanced fluctuations may be due to the atomic-site disorder on the $\mathrm{AlB}_{2}$ lattice in the present case.

\section{B. Magnetic measurements}

Measurements of magnetization $(M)$ versus temperature show the expected peak above $3 \mathrm{~K}$ corresponding to the AF transition. In Fig. 3, the data are plotted as $(1 / M)$ to show the Curie-Weiss behavior. In light of the CEF splittings obtained from the heat capacity, paramagnetic behavior for $J$ $=9 / 2$ local moments should be observed well above the temperature corresponding to the largest splitting energy $(146 \mathrm{~K})$. Such a fit above $240 \mathrm{~K}$ yields a negative Curie temperature $\theta_{N}=-12 \mathrm{~K}$ and an effective moment $p_{\text {eff }}=3.62$. The latter is identical to the free-ion moment for Nd, showing that the magnetic moment resides in $\mathrm{Nd}$ local moments. Note that by extending the fitting range down to $80 \mathrm{~K}$, we obtained a somewhat reduced value, $\theta_{N}=-7.5 \mathrm{~K}$, identical to the previous result, ${ }^{6}$ however, it is now clear that CEF splitting of $\mathrm{Nd}$ levels comes into play for this temperature range.

Below $10 \mathrm{~K}, 1 / M$ drops off due to the nearness of the magnetic transition. However, for a narrow range of 14-25 K, Curie-Weiss behavior is also observed, with a least-square fit yielding $p_{\text {eff }}=3.16$, with a small positive paramagnetic Curie temperature. The small positive Curie temperature associated with an AF transition is similar to the result ${ }^{6}$ for $\mathrm{PrCuGe}$ and could result from competing interactions in different crystallographic directions. The fitted curve is shown by a second straight line in the figure. For a groundstate CEF doublet composed of levels $\pm m_{J}, p_{\text {eff }}$ will be $2 g m_{J} \sqrt{3 / 4}$. Taking $g=8 / 11$, which is the Landé $g$ factor for $J=9 / 2 \mathrm{Nd}$, the observed $p_{\text {eff }}=3.16$ yields $m_{J}=2.51$, indicating that the ground-state doublet is likely composed of pure $\pm 5 / 2$ levels, with $m_{J} \equiv 2.5$. With this as a basis, we found very satisfactory agreement above the magnetic transition, using CEF values from the heat capacity with no additional parameters, by assuming that the ground-state doublet is composed of $\pm 5 / 2$ levels and that the $65 \mathrm{~K}$ levels are $\pm 3 / 2$ and $\pm 7 / 2$ and the $146 \mathrm{~K}$ levels are $\pm 1 / 2$ and $\pm 9 / 2$. The

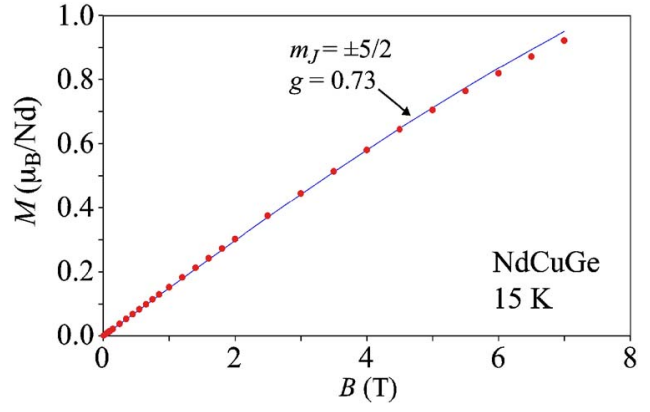

FIG. 4. (Color online) Magnetization vs field up to $7 \mathrm{~T}$ for $\mathrm{NdCuGe}$ at $15 \mathrm{~K}$. Solid curve: Brillouin-function fit for $g$ and $J_{z}$ as labeled.

magnetization resulting from this model is shown by the solid curve in Fig. 3, which nearly coincides with the the straight line fits except in the cross-over region, as indicated in the inset. Note that for the CEF-based curve, no magnetic interactions (hence, no $\theta_{N}$ ) were included.

Additional information is provided by $M$ versus $H$ curves, shown in Figs. 4 and 5. At 15 K (Fig. 4), a Brillouin function corresponding to a doublet of $\pm 5 / 2$ levels (solid curve) quite closely follows the data. For this temperature and range of fields, occupation of the CEF-split levels is negligible so that the magnetic state at low temperatures can be accurately regarded as being built from the $\pm 5 / 2$ groundstate levels only. At $2 \mathrm{~K}$, saturation is incomplete in a field of $7 \mathrm{~T}$ (Fig. 5), however, a fitted extrapolation function of the form $^{14} M=A / H+B / H^{2}$ yielded a saturation moment of $1.88 \mu_{B}$. This is nearly identical to the value $g m_{J}=(8 / 11)$ $\times(5 / 2)=1.82$ expected for a magnetic state composed of $\pm 5 / 2$ levels. This saturation moment is somewhat larger than the value (1.24) deduced from neutron scattering ${ }^{6}$ at $1.6 \mathrm{~K}$, the difference likely resulting from a combination of thermal agitation and local variations in moment orientation due to site disorder in $\mathrm{NdCuGe}$.

\section{Resistivity measurements}

The electrical resistivity of $\mathrm{NdCuGe}$ depends on temperature, as shown in Fig. 6. At higher temperatures, it behaves as does a normal metal, proportional to the temperature, and likely dominated by the electron-phonon interaction. In an intermediate temperature range of $10-60 \mathrm{~K}$, there is a downward concave form, a reduction in scattering which may be attributed to CEF effects. At lower

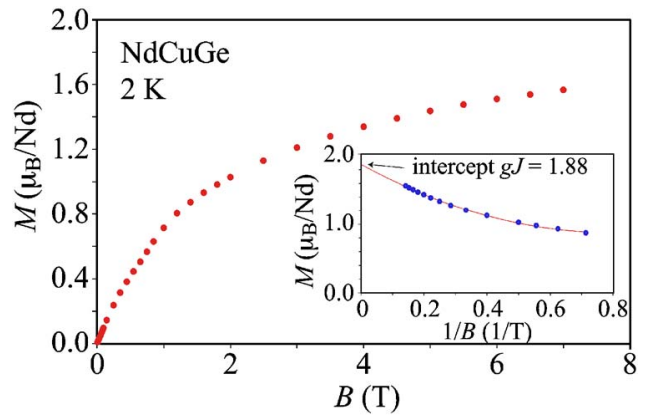

FIG. 5. (Color online) Magnetization vs field up to $7 \mathrm{~T}$ for $\mathrm{NdCuGe}$ at $2 \mathrm{~K}$. Inset: fit yielding saturation moment shown. 


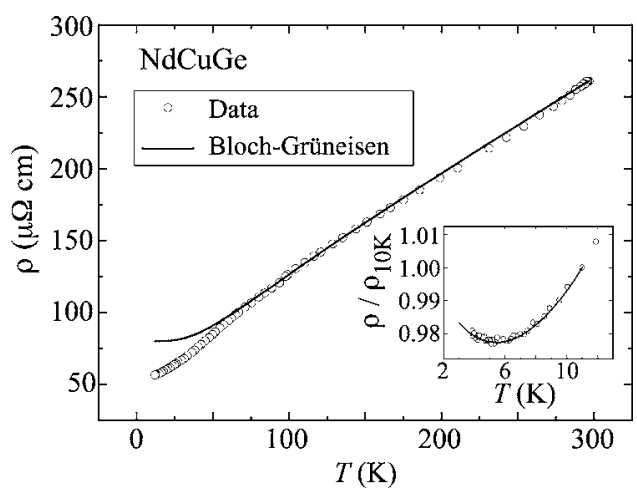

FIG. 6. Electrical resistivity vs temperature. Continous curve: fit of BG equation to high-temperature experimental data. Inset: expanded lowtemperature data, showing shallow minimum near $5.7 \mathrm{~K}$; curve is guide to the eye.

temperatures, there is a shallow minimum (see lower inset to Fig. 6). The upturn is reminiscent of spin-glass behavior ${ }^{15}$ and may be related to the slow freeze-out of magnetic fluctuations seen above $T_{N}$ in the heat capacity measurements. This could be due to individual spins that do not order at $T_{N}$ due to the random site disorder. Judging from the entropy associated with this tail (Fig. 2), it is possible that as many as $10 \%$ of the spins are involved, although it is difficult to determine this with any precision.We also observed a small irreversibility in the field-cooled versus zero-field-cooled magnetization curves in this range, below $20 \mathrm{~K}$. Note that $T_{N}$ is below the range of the resistivity measurements. The resistivity at room temperature is about $260 \mu \Omega \mathrm{cm}$. This is large for a metallic system although still in the metallic range. This may be due in part to a large electron-phonon interaction, which is consistent with the large phonon-drag term in thermopower (see below). The resistivity for $\mathrm{CeCuGe}$ is also relatively large, ${ }^{4}$ with a room temperature value approximately two times smaller than that of $\mathrm{NdCuGe}$.

To analyze these data further, and assuming that Matthiessen's rule holds, we express the electrical resistivity in terms of four major contributions,

$$
\rho(T)=\rho_{0}+\rho_{\mathrm{ph}}(T)+\rho_{\mathrm{sf}}(T)+\rho_{\mathrm{CEF}},
$$

where $\rho_{0}$ is the residual resistivity, $\rho_{\mathrm{ph}}$ is the lattice or phonon contribution, and $\rho_{\mathrm{sf}}$ and $\rho_{\mathrm{CEF}}$ are magnetic contributions associated with electron-spin fluctuation scattering and spindependent scattering between CEF-split levels, respectively. For the phonon contribution, we used the Bloch-Gruneisen (BG) equation,

$$
\rho_{\mathrm{ph}}=\rho_{\mathrm{ph}}^{0}+4 R T\left(T / \Theta_{D}\right)^{4} J_{5}\left(\Theta_{D} / T\right),
$$

where $\Theta_{D}$ is the Debye temperature, $J_{5}\left(\Theta_{D} / T\right)$ is the Grüneisen function, $R$ refers to the linear dependence at high temperature, and $\rho_{\mathrm{ph}}^{0}$ is an additive constant which includes the residual resistivity plus the other terms in Eq. (1), which are changing at low temperatures. The solid curve in Fig. 6 shows the BG fit to the data. The fitting parameters $R, \rho_{\mathrm{ph}}^{0}$, and $\Theta_{D}$ are found to be $2.53 \mu \Omega \mathrm{cm} / \mathrm{K}, 80 \mu \Omega \mathrm{cm}$, and $242 \mathrm{~K}$, respectively. The obtained value, $\Theta_{D}=242 \mathrm{~K}$ is very close to the range of values, $\Theta_{D}=240-260 \mathrm{~K}$ obtained from the heat capacity measurements over this temperature range.
We assume for the resistivity analysis that the Debye temperature remains constant in the temperature range of $10-100 \mathrm{~K}$ and, hence, the temperature dependence of the lattice resistivity ${ }^{16}$ is $\left(\rho_{\mathrm{ph}}-\rho_{\mathrm{ph}}^{0}\right) \sim\left(T / \Theta_{D}\right)^{5}$. This holds to an accuracy of $0.1 \%$ at low temperatures.

Considering the magnetic contribution as the last two terms in Eq. (1), $\rho_{\text {mag }}=\rho_{\text {sf }}+\rho_{\mathrm{CEF}}, \rho_{\text {sf }}$ generally behaves ${ }^{17}$ as $A T^{2}$, while the spin-disorder term $\rho_{\mathrm{CEF}}$ depends on crystalfield-split level populations, which can give rise to further temperature dependence ${ }^{18,19}$ for $\rho_{\text {mag }}$. The latter is temperature independent at higher temperatures. For the theoretical calculation, we used the following expression: ${ }^{18,19}$

$\rho_{\mathrm{CEF}}(T)=\frac{3 \pi N m}{\hbar e^{2} E_{F}} G^{2}(g-1)^{2} \sum_{m_{s}, m_{s^{\prime}}, i, i^{\prime}}\left\langle m_{s^{\prime}}, i^{\prime}|\vec{s} \cdot \vec{J}| m_{s}, i\right\rangle^{2} p_{i} f_{i i^{\prime}}$,

where $m_{s}$ and $m_{s^{\prime}}$ are the conduction electron spins in the initial and final states and the matrix elements join the local moment and conduction electron systems. $G$ is a parameter related to the exchange coupling. The $f_{i i^{\prime}}$ in the equation are given by

$$
f_{i i^{\prime}}=\frac{2}{1+\exp \left(-E_{i i^{\prime}} / k_{B} T\right)},
$$

where $E_{i i^{\prime}}$ is the energy difference between $E_{i}$ for state $i$ and $E_{i^{\prime}}$ for state $i^{\prime}$. The probability $p_{i}$ that an electron is in state $i$ with the energy $E_{i}$ is given by the Boltzmann relation

$$
p_{i}=\frac{N_{i}}{N}=\frac{\exp \left(-E_{i} / k_{B} T\right)}{\sum_{j} \exp \left(-E_{j} / k_{B} T\right)},
$$

with the sum taken over all CEF-split states. According to the magnetic analysis, we used a ground-state doublet composed of $J_{z}= \pm 5 / 2$ levels and the corresponding $g$ value $g$ $=8 / 11$. We found that a first excited $( \pm|3 / 2\rangle, \pm|7 / 2\rangle)$ quartet and a second excited a $( \pm|1 / 2\rangle, \pm|9 / 2\rangle)$ quartet gave good agreement with the data. This is the same level scheme that provided good agreement for the temperature dependence of the dc susceptibility. Using Eqs. (3)-(5), this leads to the following expression:

$$
\begin{aligned}
\rho_{\mathrm{CEF}}= & \frac{3 \pi N m}{\hbar e^{2} E_{F}} G^{2}(g-1)^{2} p_{i}(6.25+14.5 x+23.625 y \\
& \left.+18.5 \frac{x}{x+1}+16.5 \frac{x y}{x+y}\right),
\end{aligned}
$$

with $x=\exp \left(-\Delta_{1} / T\right), y=\exp \left(-\Delta_{2} / T\right), p_{i}=1 / 1+2 x+2 y, \Delta_{1}$ is the spitting energy between the ground state and the lower quartet, and $\Delta_{2}$ is the separation between the ground state and the upper quartet. At temperatures comparable to $\Delta_{1}$ and $\Delta_{2}$, in other words, as $x \rightarrow 1$ and $y \rightarrow 1$, the quantity inside the parenthesis tends to the value of 61.875 , which is $(5 / 2) J(J+1)$ for $J=9 / 2$ and $p_{i}=1 / 5$ so that the above equation in this limit becomes identical to the temperature independent values of $\rho_{\mathrm{CEF}}$.

Subtracting $\rho_{\mathrm{ph}}$ from the raw resistivity data, we fit $\Delta \rho_{\text {mag }}=\rho-\rho_{\mathrm{ph}}$ according to the above expressions, with the four parameters $\Delta_{1}, \Delta_{2}, A$ (the prefactor of $\rho_{\mathrm{sf}}$ ), and the pref- 


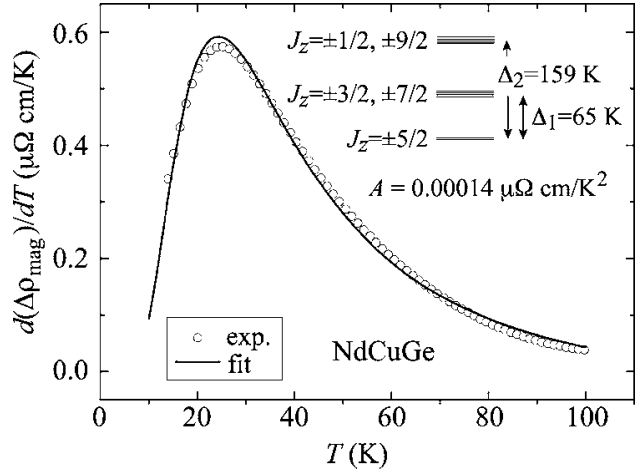

FIG. 7. Temperature derivative of electrical resistivity ( $\rho_{\text {mag }}$, with BlochGruneisen fit removed), plotted vs $T$, and theoretical curve based on CEF model described in the text. Fitted CEF parameters are also illustrated, along with fitted $T^{2}$ coefficient $A$.

actor of $\rho_{\mathrm{CEF}}$. To increase the accuracy and quality of fitting, we used the temperature derivative of $\Delta \rho_{\mathrm{mag}}$. Figure 7 shows a plot of $d \Delta \rho_{\mathrm{mag}} / d T$ versus $T$, along with the fit (solid curve). The best-fit values yield $\Delta_{1}=65 \mathrm{~K}, \Delta_{2}=159 \mathrm{~K}, \quad A$ $=0.00014 \mu \Omega \mathrm{cm} / \mathrm{K}^{2}$, and $\rho_{\mathrm{CEF}}^{0}=28 \mu \Omega \mathrm{cm}$, where the latter is the $T=0$ value of Eq. (6), corresponding to $x=y=0$. Note that, in principle, $\rho_{\mathrm{CEF}}^{0}$ should be included to $\rho_{0}$ in Eq. (1); however, since we fit to the derivative of the resistivity, the nonmagnetic portion of $\rho_{0}$ was not determined in this fit. A very good fitting was obtained over the entire temperature range where magnetic scattering dominates and the CEF splittings obtained are in good agreement with the heat capacity analysis.

\section{Thermoelectric power}

The thermoelectric power $(S)$ is of particular interest because of its sensitivity to variations of the density of states near the Fermi energy. The temperature dependence of $S$ is plotted in Fig. 8. The general behavior is similar to what is expected in metals, with a $T^{3}$ behavior at low temperatures indicative of a phonon-drag mechanism, changing to asymptotically linear behavior at high temperatures. The positive sign for $S$ at high temperature shows that hole conduction dominates. This is in contrast to that of $\mathrm{CeCuGe}$ in which $S$ passes through a maximum at about $30 \mathrm{~K}$ and eventually

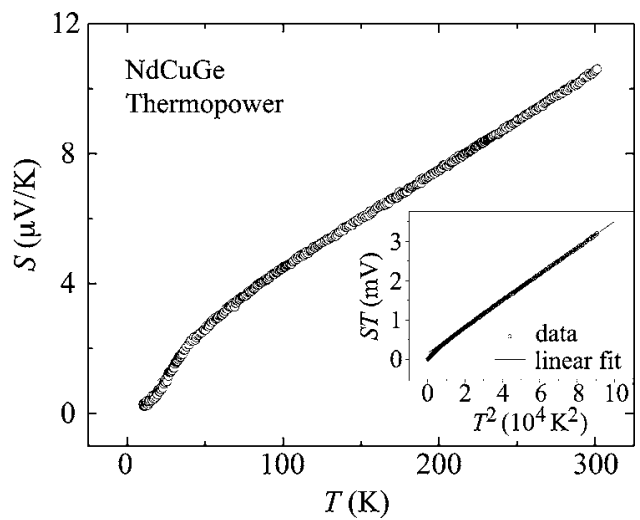

FIG. 8. Seebeck coefficient $S$ vs $T$. Inset: $S T$ vs $T^{2}$ and fitted curve illustrating $S=A T+B / T$ behavior at higher temperatures.



FIG. 9. $S / T$ vs $T$ for NdCuGe. Also shown: $S=\alpha T+\beta T^{3}$ fit at lower temperatures and fitting parameters.

changes sign to negative at higher temperatures, ${ }^{4}$ with $T^{2}$ behavior also observed in the range of $10-30 \mathrm{~K}$, presumably due to strong spin fluctuation effects.

For $\mathrm{NdCuGe}$, the quantity $S / T$ versus $T^{2}$ is plotted in Fig. 9. There are two distinct temperature regions, with a cusp appearing between them at about $T=40 \mathrm{~K}$. The lowtemperature region, with $S / T$ nearly linear in $T^{2}$, follows the standard behavior, ${ }^{20} S=\alpha T+\gamma T^{3}$, with the first term an electronic diffusion contribution and the second typically associated with phonon drag. The coefficient $\gamma$ is in the range observed for alloys. ${ }^{20}$ As for the high-temperature region, above $140 \mathrm{~K}, S T$ versus $T^{2}$ plot gives a linear fit as expected for metals (inset of Fig. 8). The best fit according to the classical behavior $S=A T+B / T$ gives $B=160 \mu \mathrm{V}$ and $A$ $=0.0334 \mu \mathrm{V} / \mathrm{K}^{2}$, with the positive sign for the latter contrasting the behavior of $\mathrm{CeCuGe}{ }^{4}$

The large difference between linear- $T$ contributions for $\mathrm{NdCuGe}$ and $\mathrm{CeCuGe}$ is surprising since the rare earth exhibits the same $3+$ valence for both materials, hence, the electronic structures might be expected to be similar. We deduce that the Fermi surface must be near a minimum or a sharp feature in the density of states since large changes result even for isoelectronic substitution. At intermediate temperatures, $\mathrm{CeCuGe}$ exhibits a prominent peak $^{4}$ in $S(T)$, while no peak is observed for $\mathrm{NdCuGe}$. Extending the fitted high-temperature line $S=A T$ to low temperatures, the difference curve does exhibit a very broad maximum in the range of 50-100 K, approximately ten times lower in peak magnitude than that of $\mathrm{CeCuGe}$. Thus, the lack of obvious peak in $\mathrm{NdCuGe}$ can be attributed to the large slope $A T$ combined with a relatively modest phonon-drag contribution, while the larger feature in $\mathrm{CeCuGe}$ can be attributed to scattering from CEF-split levels of the localized magnetic ions. For such a mechanism, ${ }^{21,22}$ a prominent peak occurs at a temperature somewhat smaller than the CEF splitting and is largest when the resonant exchange scattering and elastic potential scattering terms are comparable in magnitude. $\mathrm{NdCuGe}$ exhibits an exchange scattering term, which contributes to the resistivity, as shown in Eq. (3). This term should also contribute to the thermopower, although the exchange term will be considerably smaller for $\mathrm{Nd} f$ levels that were further separated from $E_{F}$, as was established within the Anderson model. ${ }^{21}$ Thus, the broadened feature in $S(T)$ for $\mathrm{NdCuGe}$ near $50-100 \mathrm{~K}$ is 
due to phonon drag possibly enhanced by scattering from CEF-split levels. In $\mathrm{CeCuGe}$, the latter term is significantly larger, while the overall difference in $S(T)$ for the two materials points to a narrow pseudogaplike feature in the density of states.

\section{CONCLUSIONS}

We have carried out magnetization, magnetic susceptibility, electrical resistivity, and thermoelectric power measurements on $\mathrm{NdCuGe}$. The magnetization is dominated by $\mathrm{Nd}^{3+}$ ionic moments at high temperatures, while at low temperatures, CEF splitting of the rare-earth levels comes into play. With a combination of heat capacity, magnetization, and resistivity, we obtained a consistent estimation of the CEF splitting energies and show that the ground state is composed of a $J_{z}= \pm 5 / 2$ doublet. Saturation measurements show that the previously observed low-temperature magnetic state is formed from this doublet. The resistivity could be fit according to a model including spin-disorder scattering from CEF-split levels, plus a spin fluctuation term $\left(A T^{2}\right)$, with the coefficient $A$ nearly two orders of magnitude smaller than previously observed in isostructural $\mathrm{CeCuGe}$. The near absence of the latter term in $\mathrm{NdCuGe}$ indicates that spin fluctuation or $e-e$ scattering is not significant in this material. Similarly, the $\mathrm{NdCuGe}$ thermopower could be fit to classic metallic behavior, with a much smaller CEF-scattering term than for $\mathrm{CeCuGe}$, indicating the $\mathrm{Nd}$ analog to behave as a simple metal without the large spin fluctuation effects apparent in $\mathrm{CeCuGe}$. The high-temperature limits correspond to different carrier types, pointing to a likely narrow pseudogap in the density of states near $E_{F}$.

\section{ACKNOWLEDGMENTS}

This work was supported by the Robert A. Welch Foundation (Grant No. A-1526) and the National Science Foundation (DMR-0315476).

${ }^{1}$ A. Szytula and J. Leciejewicz, Handbook of Crystal Structures and Magnetic Properties of Rare Earth Intermetallics (CRC, Boca Raton, FL, 1994).

${ }^{2}$ A. Szytula, D. Fus, B. Penc, and A. Jezierski, J. Alloys Compd. 317-318, 340 (2001).

${ }^{3}$ C. P. Sebastian, H. Eckert, C. Fehse, J. P. Wright, J. P. Attfield, D. Johrendt, S. Rayaprol, R.-D. Hoffmann, and R. Pottgen, J. Solid State Chem. 179, 2376 (2006).

${ }^{4}$ Y. Oner, O. Kamer, J. H. Ross, Jr., C. S. Lue, and Y. K. Kuo, Solid State Commun. 136, 235 (2005).

${ }^{5}$ F. Yang, J. P. Kuang, J. Li, E. Bruck, H. Nakotte, F. R. de Boer, X. Wu, Z. Li, and Y. Wang, J. Appl. Phys. 8, 4705 (1991).

${ }^{6}$ S. Baran, A. Szytula, J. Leciejewicz, N. Stusser, A. Zygmunt, Z. Tomkowicz, and M. Guillot, J. Alloys Compd. 243, 112 (1996).

${ }^{7}$ A. Iandelli, J. Alloys Compd. 198, 141 (1993).

${ }^{8}$ C. S. Lue and Y. K. Kuo, J. Phys.: Condens. Matter 15, 877 (2003).

${ }^{9}$ E. S. R. Gopal, Specific Heats at Low Temperatures (Plenum, New York, 1966).

${ }^{10}$ M. Bouvier, P. Lethuillier, and D. Schmitt, Phys. Rev. B 43, 13137 (1991).

${ }^{11}$ Crystal Field Handbook, edited by D. J. Newman and B. Ng (Cambridge University Press, 2000).

${ }^{12}$ A. Tari, The Specific Heat of Matter at Low Temperatures (Imperial College Press, London, 2003).

${ }^{13}$ V. Goruganti, Y. Li, J. H. Ross, Jr., K. D. D. Rathnayaka, and Y. Oner, J. Appl. Phys. 99, 08P303 (2006).

${ }^{14}$ S. Chikazumi and C. D. Graham, Physics of Ferromagnetism (Oxford University Press, Oxford, 1997).

${ }^{15}$ D. X. Li, Y. Shiokawa, Y. Homma, A. Uesawa, A. Dönni, T. Suzuki, Y. Haga, E. Yamamoto, T. Honma, and Y. Ōnuki, Phys. Rev. B 57, 7434 (1998).

${ }^{16}$ J. M. Ziman, Electrons and Phonons (Clarendon, Oxford, 2001).

${ }^{17}$ P. L. Rossiter, The Electrical Resistivity of Metals (Cambridge University Press, Cambridge, 1991).

${ }^{18}$ V. U. S. Rao and W. E. Wallace, Phys. Rev. B 2, 4613 (1970).

${ }^{19}$ Y. Oner, O. Kamer, E. Alveroglu, M. Acet, and T. Krenke, J. Alloys Compd. 429, 64 (2007).

${ }^{20}$ F. Blatt, P. Schroeder, C. Foiles, and D. Greig, Thermoelectric Power of Metals (Plenum, New York, 1976).

${ }^{21}$ A. K. Bhattacharjee and B. Coqblin, Phys. Rev. B 13, 3441 (1976).

${ }^{22}$ V. Zlatić, B. Horvati, I. Milat, B. Coqblin, G. Czycholl, and C. Grenzebach, Phys. Rev. B 68, 104432 (2003). 